\title{
A Novel Scheme for NLOS Identification using Energy Detector in 60 GHz Systems
}

\author{
Xiaolin Liang ${ }^{1}$, Hao Zhang ${ }^{1}{ }^{2}$, Tingting $\mathrm{Lv}^{1}$, Xuerong Cui ${ }^{3}$ and T. Aaron. Gulliver ${ }^{2}$ \\ ${ }^{1}$ College of Information Science and Engineering, Ocean University of China, \\ Qingdao, 266100, China \\ ${ }^{2}$ Department of Electrical and Computer Engineering, University of Victoria, \\ Victoria, V8W 3P6, Canada \\ ${ }^{3}$ Department of Computer and Communication Engineering, China University of \\ Petroleum (East China), Qing Dao, China \\ xiaolin87liang@163.com, zhanghao@ouc.edu.cn, \\ lvtingting33@163.com,cuixuerong@163.com \\ agullive@ece.uvic.ca
}

\begin{abstract}
The major problem of indoor localization is presence of non-line-of-sight (NLOS) channels. In order to perform NLOS identification, in this paper, we propose a novel NLOS identification technique based on minimum slope and standard deviation of energy block of the received signal using energy detector. In particular, IEEE $802.15 .3 \mathrm{c} 60 \mathrm{GHz}$ channel models are used as examples and above statistics is found to be explained in detail. The simplicity of the proposed approach lies in use of parameters of energy-based time of arrival (TOA) estimation algorithm. CM1 (LOS) and CM2 (NLOS) channel models of IEEE 802.15.3c channel models are used. Numerical simulations results show that correct identification of channel models with the proposed approach is better than with multipath channel statistics based approach.
\end{abstract}

Keywords: NLOS identification, minimum slope, standard deviation, $60 \mathrm{GHz}$, energy detector, IEEE 802.15.3c

\section{Introduction}

The location of a mobile terminal (MT) can be estimated using different parameters of a received signal, such as TOA, angle-of-arrival (AOA), and/or the received signal strength (RSS). $60 \mathrm{GHz}$ has a great potential for accurate ranging and localization systems due to its very wide bandwidth and capability in resolving individual multipath components [1-6]. Therefore, TOA of the received signal can be estimated with high accuracy for $60 \mathrm{GHz}$ systems if the first arriving path has been identified precisely. One of the major challenges for localization systems is mitigation of NLOS effects. If direct path between a fixed terminal (FT) (An FT is usually a base station in a cellular network or an anchor node in a sensor network.) and MT is being obstructed, TOA of signal to FT will be delayed, which introduces a positive bias. Using such NLOS TOA estimates during localization of MT position may significantly degrade positioning accuracy. Hence, FTs that are under NLOS condition have to be identified and their effects have to be mitigated.

NLOS identification and mitigation techniques have been discussed extensively in the literature, but mainly within the cellular network framework [7-13]. For example, in [7], standard deviation of ranging measurements is compared with threshold for NLOS identification, where measurement noise variance is assumed to be known. In [8], a decision-theoretic NLOS identification framework is presented, where various hypothesis tests are discussed for known and unknown probability density functions of TOA 
measurements. Guvenc et al. $[9,13]$ used mean excess delay, root mean square delay, and kurtosis of multipath channel as NLOS identification metrics. When statistics of kurtosis, mean excess, and RMS delays are priori known, likelihood ratio tests can be performed for hypothesis selection. Another method was proposed by Heidari et al. [10]. They tried to find the first detected path of the received signal as a peak of filtered channel impulse radio. As similar to previous method, this technique also used joint likelihood function using mean excess delay $\tau_{\text {med }}$, total received power $P_{t o t}$ and hybrid of power of the first detected path and TOA of first detected path $\xi_{h y b}$.

Venkatesh et al. [11] identified channel based on TOA, RSS, and RMS delay spread of the received signal. The conditional distributions of TOA, RSS, and RDS estimates are functions of distance and channel state. Provided that physical distance between transmitted and receive nodes is known exactly, state of channel can be identified by comparing likelihood values for each of estimates (TOA, RSS, and RDS), conditioned on distance. Shimizu et al. [12] performed intensive measurements of path-loss and delay-profile characteristics of LOS and NLOS environments in a suburban residential area. Based on their analysis, they found that the delay spread was dependent on distance, and NLOS delay spread was found to be several times larger than that of LOS case. The standard deviation of delay spread for NLOS cases ranged from 80 to $200 \mathrm{~ns}$, which was an order of magnitude larger than that of LOS case.

In this paper, we propose a new LOS/NLOS identification approach for $60 \mathrm{GHz}$ signal, which is based on minimum slope and standard deviation of energy block of received signal using energy detector. Firstly, we use energy detector TOA Estimation algorithm for estimation TOA. Secondly, we characterize the minimum slope and standard deviation of energy block of the received signal. Finally, we use a threshold test for LOS/NLOS identification.

The remainder of this paper is organized as follows. Section II describes signal and channel model. Section III describes LOS/NLOS identification approach and Section IV presents results of numerical simulations. The concluding remarks are given in section $\mathrm{V}$.

\section{System Model}

Currently, there are two important standards that have been developed for $60 \mathrm{GHz}$ wireless communications systems, IEEE 802.15.3c and IEEE 802.11ad [14-15]. In this paper, channel models in IEEE 802.15. 3c standard are used because it is specifically designed for wireless personal area networks and thus encompasses typical indoor environments. Further, these are the most widely employed models for $60 \mathrm{GHz}$ systems. IEEE 802.15.3c standard was the first developed for high data rate short-range wireless systems. The physical layer was designed to support transmission of data within a few meters at a minimum data rate of $2 \mathrm{Gbps}$. These models have been developed for communications in frequency band 57 to $66 \mathrm{GHz}$ in indoor residential, indoor office and library environments (with differences largely due to LOS and NLOS characteristics) [16-20].

In this paper, a Pulse Position Modulation Time Hopping $60 \mathrm{GHz}$ signal is employed for ranging purposes. The propagation delay $\hat{\tau}$, between transmitter and receiver is estimated for use in localization.

\section{1. $60 \mathrm{GHz}$ Signal}

$60 \mathrm{GHz}$ signals have a very short duration (typically 100 picoseconds or less), and can be expressed as:

$$
s(t)=\sum_{-\infty}^{\infty} p\left(t-j T_{s}-C_{j} T_{c}-a_{j} \varepsilon\right)
$$


where $T_{s}$ is symbol time. The Time Hopping (TH) code represented by $C$ is a pseudorandom integer-valued sequence which is unique for each user to limit multiple access interference, and $T_{c}$ is chip time. PPM time shift is $\varepsilon$ so that if $a_{j}$ is 1 , signal is shifted in time by $\varepsilon$, while if $a_{j}$ is 0 , there is no shift. Many pulse shapes have been proposed for $60 \mathrm{GHz}$ systems. In this paper a Gaussian pulse is employed which is multiplied by the carrier signal to give:

$$
p(t)=\frac{\sqrt{2}}{\alpha} \exp \left(-2 \pi \frac{t^{2}}{\alpha^{2}}\right) \cos \left(2 \pi f_{c} t\right)
$$

where $\alpha$ is shape factor and $f_{c}$ is carrier frequency which here is $f_{c}=60 \mathrm{GHz}$. A smaller shape factor results in a shorter duration pulse and a larger bandwidth.

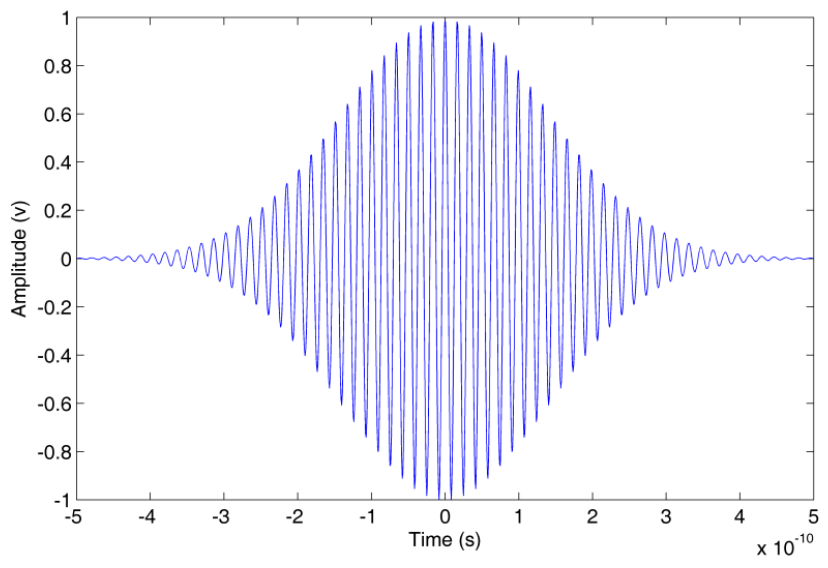

Figure 1. Waveform of $60 \mathrm{GHz}$ Signal

\subsection{Signal Shift and Path Loss}

The path loss is defined as ratio of received signal power to transmit signal power and it is very important for link budget analysis. Unlike narrow-band system, path loss for a wide-band system such as mm-wave system is both distance and frequency dependent. In order to simplify the models, it is assumed that frequency dependence path loss is negligible and only distance dependence path loss is modeled. The signal path loss, which depends on the propagation distance and the channel (IEEE802.15.3c), is described by:

$$
P L(d)[d B]=P L_{0}+10 \cdot n \log _{10}\left(\frac{d}{d_{0}}\right)+X_{\sigma}[d B] ; d \geq d_{0}
$$

where $d_{0}$ and $d$ denote reference distance, and distance respectively. $n$ is path loss exponent for mm-wave, $X_{\sigma}$ is that the unit $\mathrm{dB}$, with mean zero and variance $\sigma_{s}$ for a Gaussian random variable. Table 1 summarizes the values of $\mathrm{n}, P L_{0}, \sigma_{s}$ for different environments and scenarios.

Table 1. Values of $\boldsymbol{n}, P L_{0}, \sigma_{s}$ for different Environments and Scenarios

\begin{tabular}{llll}
\hline environments & $n$ & $P L_{0}$ & $\sigma_{s}$ \\
\hline indoor residential (LOS) & 1.53 & 75.1 & 1.50 \\
indoor residential (NLOS) & 2.44 & 86.0 & 6.20 \\
indoor office (LOS) & 1.16 & 84.6 & 5.40 \\
indoor office (NLOS) & 3.74 & 56.1 & 8.60 \\
\hline
\end{tabular}


The signal shift can be expressed as:

$$
t=d t * \text { floor }((d / c) / d t)
$$

where $d$ denotes distance between transmitter and receiver, $d t$ is sampling period and $c$ is speed of light which is $299792458 \mathrm{~m} / \mathrm{s}$ in the air.

\subsection{Multipath Fading Channel}

The received signal can be written as:

$$
r(t)=\sum_{n=1}^{N} \alpha_{n} p\left(t-\tau_{n}\right)+n(t)
$$

where $N$ is number of received multipath components, $\alpha_{n}$ and $\tau_{n}$ denotes amplitude and delay of the $n^{\text {th }}$ path respectively, $p(t)$ is the received $60 \mathrm{GHz}$ pulse and $n(t)$ is Additive White Gaussian Noise with zero mean and two sided power spectral density $\mathrm{N}_{0} / 2$. Equation (5) can be rewritten as:

$$
r(t)=s(t) * h(t)+n(t)
$$

where $s(t)$ is transmitted signal, and $h(t)$ is channel impulse response which can be expressed as:

$$
h(t, \theta)=\sum_{k=1}^{K} \sum_{l=1}^{L_{k}} \mu_{k l} \delta\left(t-T_{k}-\tau_{k l}\right) \delta\left(\theta-\theta_{k}-\omega_{k l}\right)
$$

where $\delta($.$) is dirac-delta function, K$ is number of clusters, $L_{k}$ is number of rays in the $k^{\text {th }}$ cluster, and $\mu_{k l}, \tau_{k l}$ and $\omega_{k l}$ denote complex amplitude, delay and azimuth of the $k^{\text {th }}$ ray of the $l^{t h}$ cluster, respectively. Similarly, $T_{k}$ and $\theta_{k}$ represent delay and mean AOA of the $k^{\text {th }}$ cluster.

\subsection{Energy Detector}

After amplifier, the received signals are squared, and then input to an integrator with integration period $T b$. Because of inter-frame leakage due to multipath signals, the integration duration is $3 T_{f} / 2$, so number of signal values for $\mathrm{ED}$ is $\mathrm{N}=3 T_{f} / 2 \mathrm{~Tb}$. The integrator outputs can be expressed as:

$$
z[n]=\sum_{i=1}^{N} \int_{(i-1) T_{f}+\left(c_{j}+n-1\right) T b}^{(i-1) T_{T}+\left(c_{j}+n\right) T b} r^{2}(t) d t
$$

where $n \in\{1,2, . ., N\}$ denotes sample index with respect to starting point of integration period and $N$ is number of pulses per symbol. Here, $N$ is set to 1 , so the integrator outputs are:

$$
z[n]=\sum_{i=1}^{N} \int_{\left(c_{j}+n-1\right) T b}^{\left(c_{j}+n\right) T b} r^{2}(t) d t
$$

If $z[n]$ is integration of noise only, it has a centralized Chi-square distribution, while it has a non-centralized Chi-square distribution if a signal is present. The mean and variance of the noise and signal values are given by [17] respectively.

$$
\begin{gathered}
\mu_{0}=F \sigma^{2}, \sigma_{0}=2 F \sigma^{4} \\
\mu_{e}=F \sigma^{2}+E_{n}, \sigma_{e}^{2}=2 F \sigma^{4}+4 \sigma^{2} E_{n}
\end{gathered}
$$

where $E_{n}$ is signal energy within $n^{\text {th }}$ integration period and $F$ is number of degrees of freedom given by $F=2 B T b+1$. Here $B$ is signal bandwidth. 


\section{LOS/NLOS Identification}

\subsection{Description of Parameters}

In this paper, we distinguish between LOS or NLOS scenarios by exploiting statistics of the received signal by ED. The minimum slope and standard deviation of energy block is used in order to identify the LOS and NLOS scenarios respectively. The slope of energy values is considered as a measure. These values are divided into $(\mathrm{N}-\mathrm{M}+1)$ groups, with $\mathrm{M}$ values in each group. The slope for each group is calculated using a least squares line-fit. The minimum slope can then be expressed as:

$$
M S=\min _{1 \leq n \leq N-M+1} \text { slope }\{\text { linefit }(z[n], z[n+1], \ldots \ldots, z[n+M-1])\}
$$

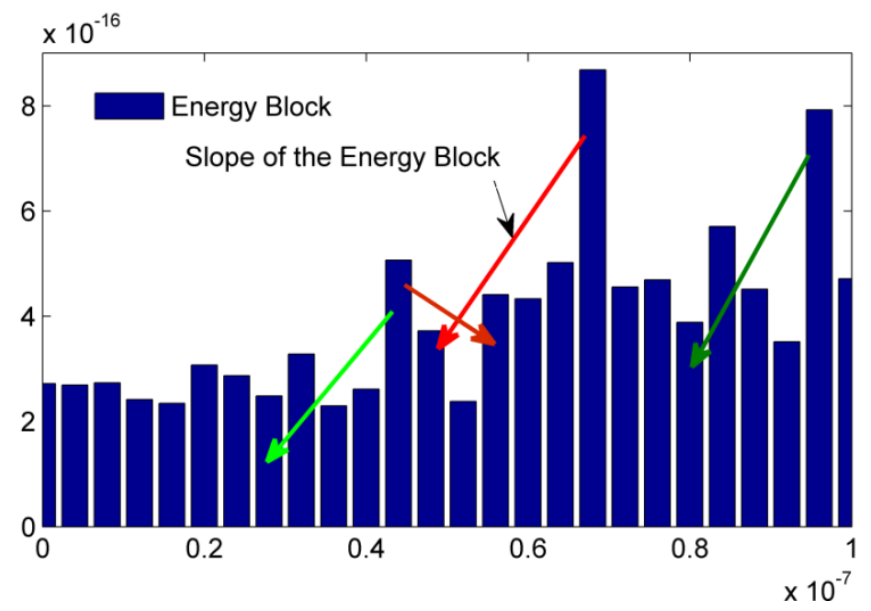

Figure 2. Slope of Energy Block

Standard deviation is a widely used measure of variability. It shows how much variation or "dispersion" there is from the average (mean or expected value). Standard deviation is given by:

\subsection{Identification Approach}

$$
D=\sqrt{\frac{\sum_{i=1}^{N}\left(x_{i}-\mu_{x}\right)^{2}}{N-1}}
$$

The ratio values of slope and standard deviation of energy block can be obtained for both LOS and NLOS scenarios using sample channel realizations from both scenarios. Here, we used sample channel realizations of IEEE 802.15.3c standard channel models in order to obtain values of slope of energy slope for both LOS and NLOS.

In order to examine characteristics of minimum slope, CM1.1 (residential LOS) and CM2.1 (residential NLOS) channel models from IEEE 802.15.3c standard are employed. For each SNR value, 1000 channel realizations are generated and sampled at $f_{c}=1 \cdot e^{10} \mathrm{~Hz}$. The other system parameters are $T_{f}=200 n s, T_{c}=1 n s$, value of $T b$ is from $1 \mathrm{~ns}$ to $4 \mathrm{~ns}$ and $N=$ 1. Each realization has a TOA uniformly distributed within $\left(0-T_{f}\right)$. Here SNR ranges from 0dB to $10 \mathrm{~dB}, 1000$ channel realizations are generated for each SNR. CM1.1 (residential LOS) and CM2.1 (residential NLOS) channel models from IEEE 802.15.3c standard are employed. Here are $11 * 1000$ samples which are got for each channel model. The relationship between minimum slope and SNR are shown in Figures 3-6. 


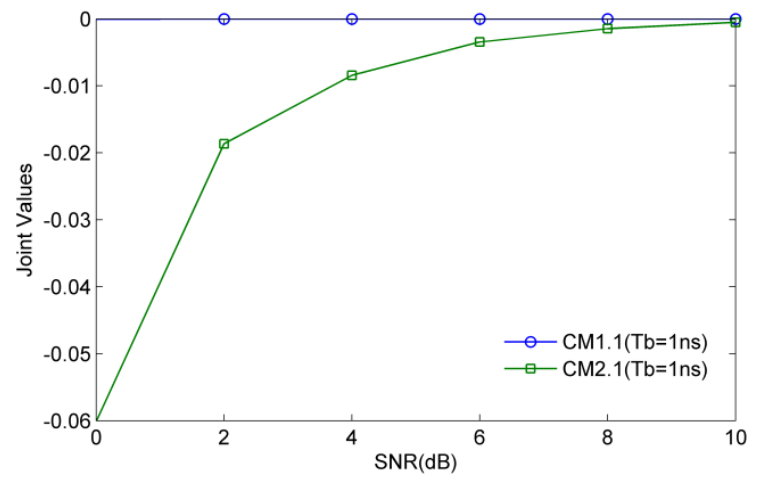

Figure 3. Joint Values with Respect to SNR (Tb=1ns)

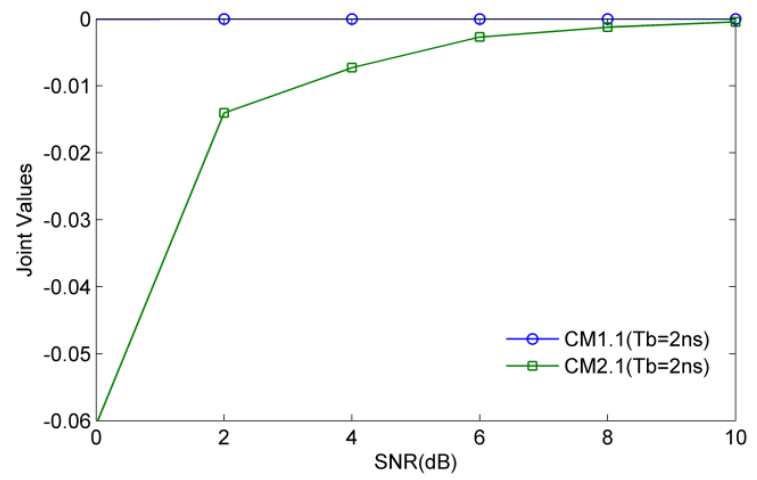

Figure 4. Joint Values with Respect to SNR $(T b=2 n s)$

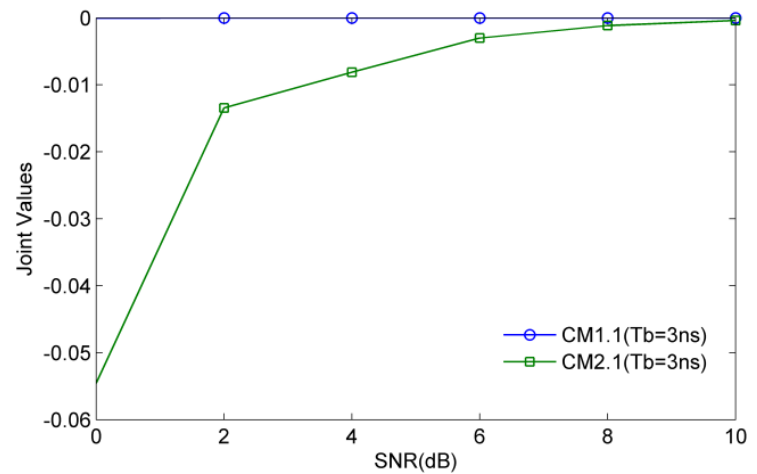

Figure 5. Joint Values with Respect to SNR (Tb=3ns)

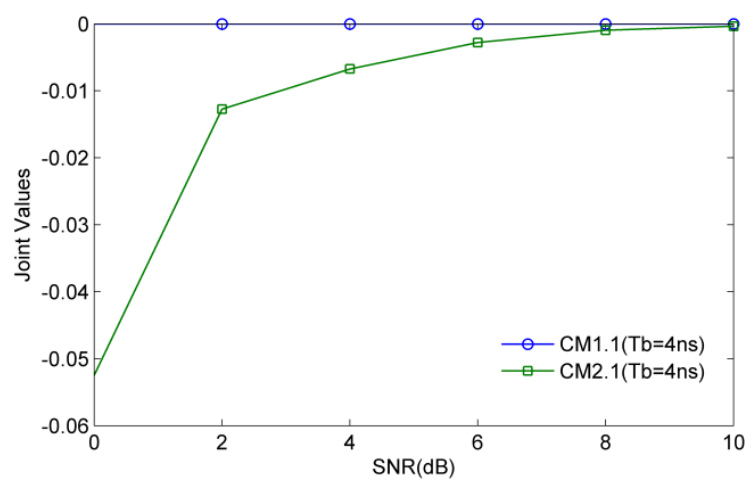

Figure 6. Joint Values with Respect to SNR $(\mathrm{Tb}=4 \mathrm{~ns})$ 
From Figures 3-6, results show that ratio values of minimum slope and standard deviation is monotonous with respect to SNR (both LOS and NLOS environment) in the $60 \mathrm{GHz}$ wireless communication system respectively. But we find minimum of joint values (LOS) is even larger than maximum of joint values of minimum slope (NLOS) when TX is 360, minimum of joint values (LOS) is even less than maximum of joint values (NLOS) when TX is others. So we propose a novel method to identify the LOS and NLOS which can be expressed as:

$$
\Theta=\alpha\left\{\begin{array}{l}
\left\{\begin{array}{l}
<\alpha_{M S} \Rightarrow N L O S \\
>\alpha_{M S} \Rightarrow L O S
\end{array} \quad \text { TX }=360^{\circ}\right. \\
\left\{\begin{array}{l}
<\alpha_{M S} \Rightarrow \text { LOS } \\
>\alpha_{M S} \Rightarrow N L O S
\end{array} \quad\right. \text { others }
\end{array}\right.
$$

where $\alpha_{M S}$ is the threshold which is chosen to identify the LOS and NLOS, TX, RX is the beam-width of measured transmitter and receiver antenna respectively. Aiming at the ranges $[\mathrm{A}, \mathrm{B}] \mathrm{dB}$ for $\mathrm{SNR}, \alpha_{M S}$ can be shown as follows :

$$
\alpha_{M S}=\max \left\{\begin{array}{l}
\operatorname{mean}\left[\operatorname{find}\left(P\left(\operatorname{abs}(M S)>\left.\Theta_{1}\right|_{A}\right) \geq \Phi\right)\right] \\
\operatorname{mean}\left[\operatorname{find}\left(P\left(\operatorname{abs}(M S)>\left.\Theta_{2}\right|_{B}\right) \geq \Phi\right)\right]
\end{array}\right\}
$$

where $\Theta_{1}$ and $\Theta_{2}$ are threshold which meet the condition $P(M S>\Theta) \geq \Phi$ where SNR is upper and lower limits. $\Phi$ is the probability value which is required for choosing suitable threshold for NLOS identification. Without loss of generality, here $\Phi$ is set to be 0.85 .

Table 2. Threshold Values for LOS and NLOS Identification

\begin{tabular}{cccccc}
\hline $\begin{array}{c}\text { Channel } \\
\text { Model }\end{array}$ & $2(\mathrm{~dB})$ & $3(\mathrm{~dB})$ & $4(\mathrm{~dB})$ & $5(\mathrm{~dB})$ & $6(\mathrm{~dB})$ \\
\hline CM1.1 & 39.0 & 45.5 & 55.6 & 60.3 & 66.6 \\
CM2.1 & 78.9 & 73.4 & 75.9 & 64.5 & 61.9 \\
CM1.2 & 92.7 & 87.1 & 78.6 & 66.2 & 59.3 \\
CM2.2 & 83.3 & 85.1 & 88.5 & 90.2 & 92.1 \\
CM1.3 & 89.7 & 82.8 & 79.0 & 68.4 & 57.3 \\
CM2.3 & 97.0 & 94.2 & 97.6 & 98.5 & 98.7 \\
CM1.4 & 90.9 & 88.2 & 80.2 & 62.5 & 60.3 \\
CM2.4 & 95.2 & 91.2 & 92.9 & 98.6 & 97.0 \\
\hline
\end{tabular}

\section{Simulated Results}

In order to verify effectiveness and practicality of algorithm, so we make a lot of simulations using IEEE 802.15.3c channel models. CM1.2, CM1.3, CM1.4 (residential LOS) and CM2.2, CM2.3, CM2.4 (residential NLOS) channel models from IEEE 802.15.3c standard are employed. For each SNR value, 1000 channel realizations are generated and sampled at $f_{c}=1 \cdot e^{10} \mathrm{~Hz}$. The other system parameters are $T_{f}=200 n s, T_{c}=1 \mathrm{~ns}$, value of $T b$ is from $1 \mathrm{~ns}$ to $4 \mathrm{~ns}$ and $N=1$. Each realization has a TOA uniformly distributed within $\left(0-T_{f}\right)$.

Here SNR ranges from $0 \mathrm{~dB}$ to $10 \mathrm{~dB}, 1000$ channel realizations are generated for each SNR. CM1.2, CM1.3, CM1.4 (residential LOS) and CM2.2, CM2.3, CM2.4 (residential NLOS) channel models from IEEE 802.15.3c standard are employed. Here are $6 * 1000$ samples which are got for each channel model. The relationship between joint parameter and SNR are shown in Figures 7-18. 


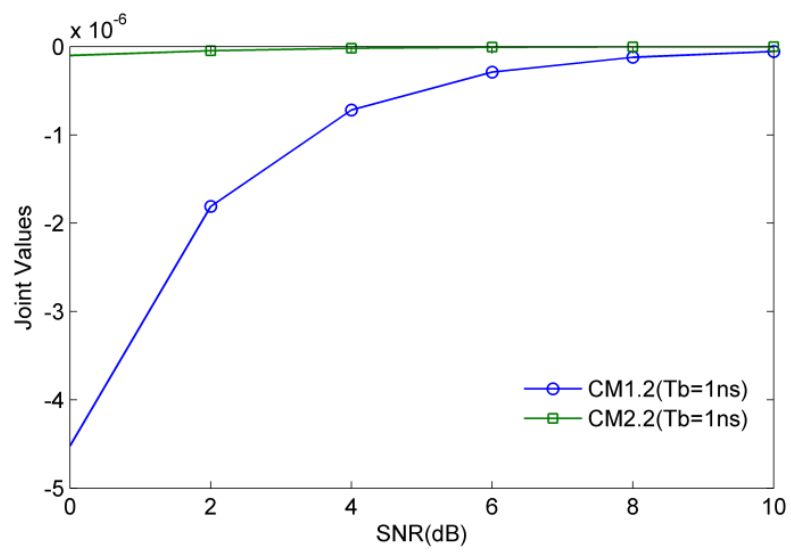

Figure 7. Joint Values with Respect to SNR (Tb=1ns)

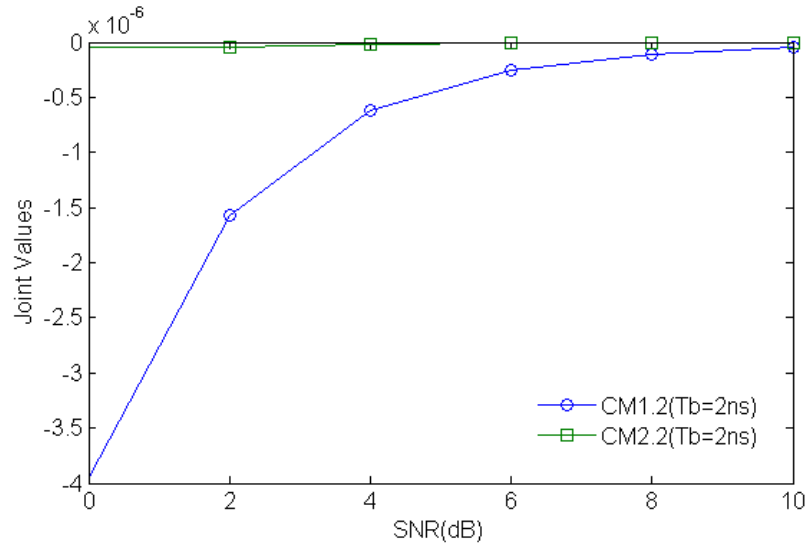

Figure 8. Joint Values with Respect to SNR $(\mathrm{Tb}=2 \mathrm{~ns})$

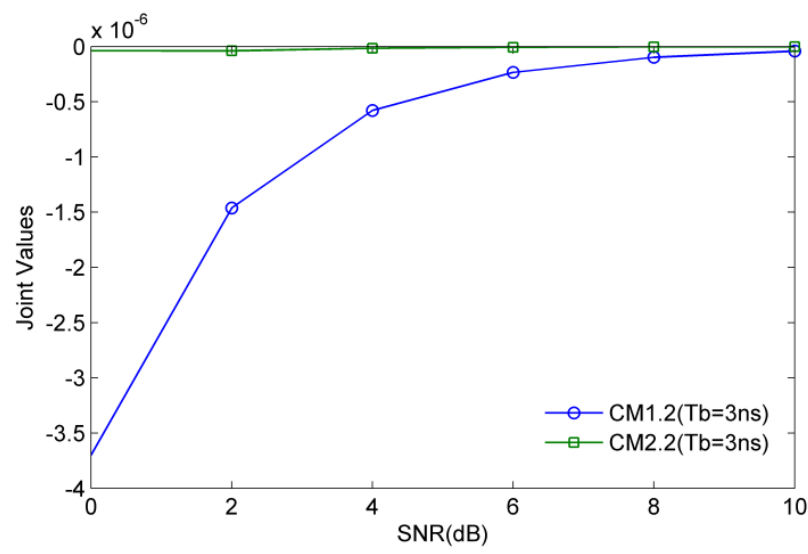

Figure 9. Joint Values with Respect to SNR $(\mathrm{Tb}=3 \mathrm{~ns})$ 


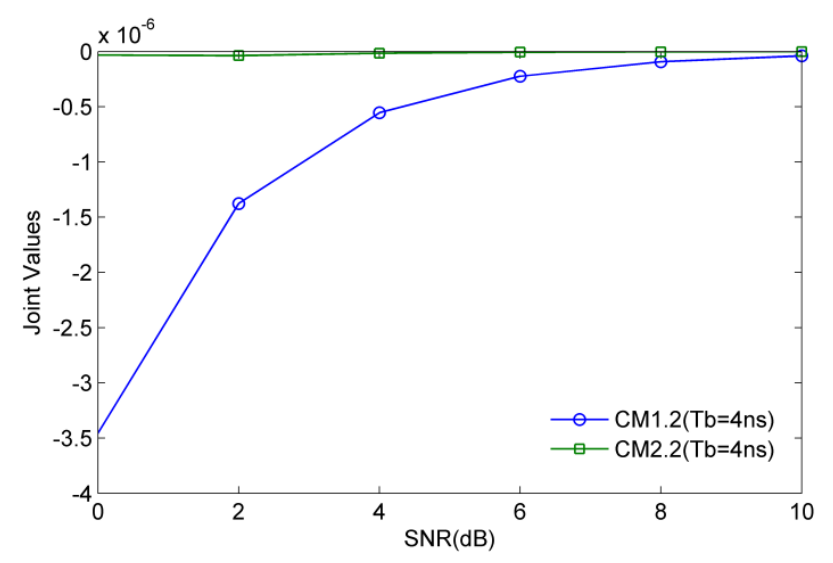

Figure 10. Joint Values with Respect to SNR (Tb=4ns)

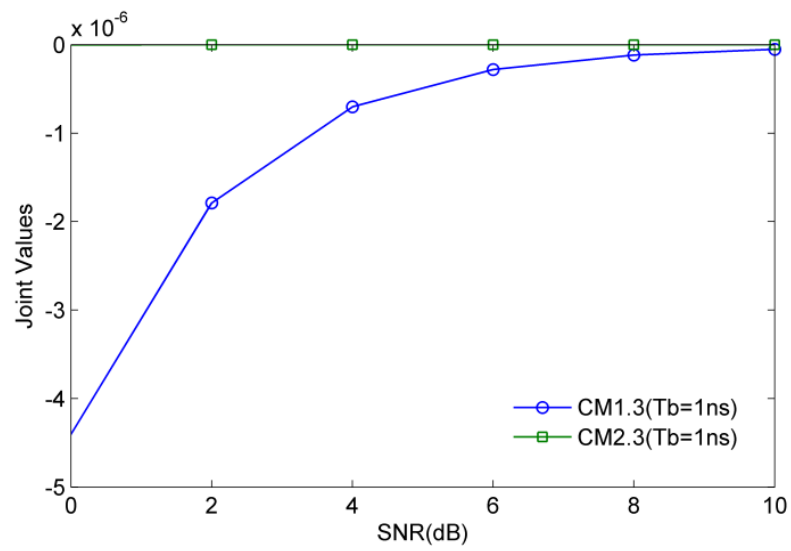

Figure 11. Joint Values with Respect to SNR ( $T b=1 \mathrm{~ns})$

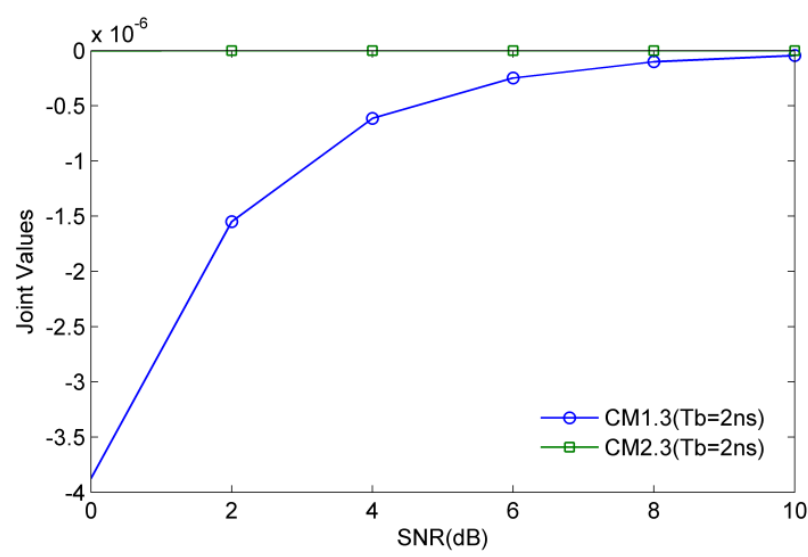

Figure 12. Joint Values with Respect to SNR ( $T b=2 n s)$ 


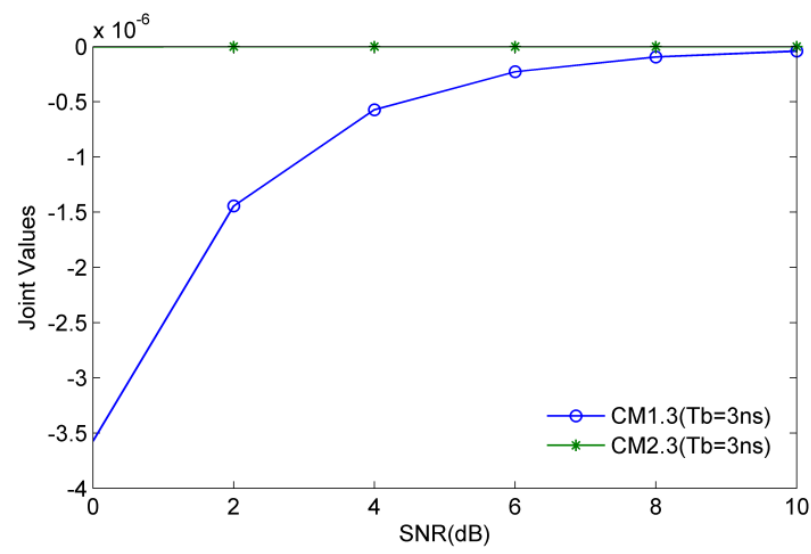

Figure 13. Joint Values with Respect to SNR ( $T b=3 n s)$

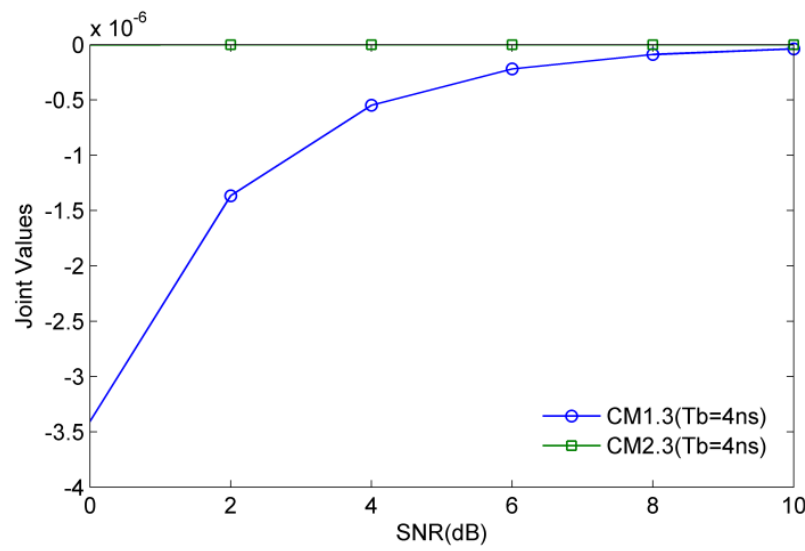

Figure 14. Joint Values with Respect to SNR ( $T b=4 n s)$

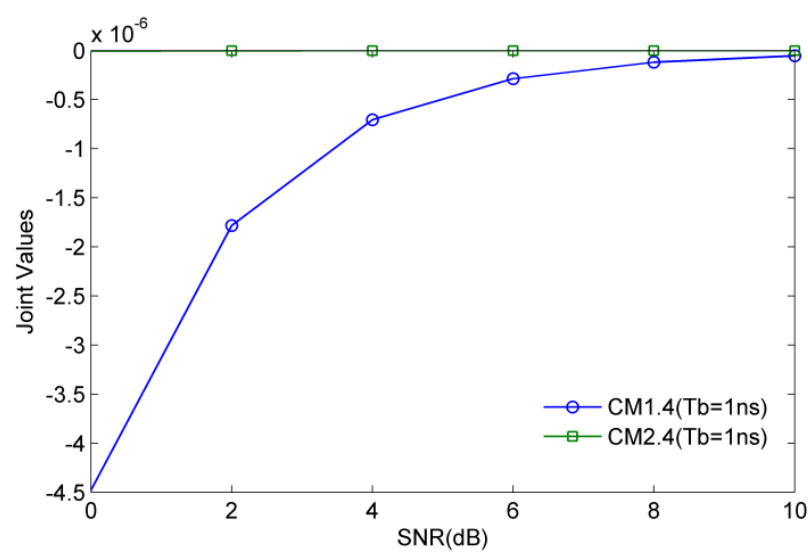

Figure 15. Joint Values with Respect to SNR ( $T b=1 n s)$ 


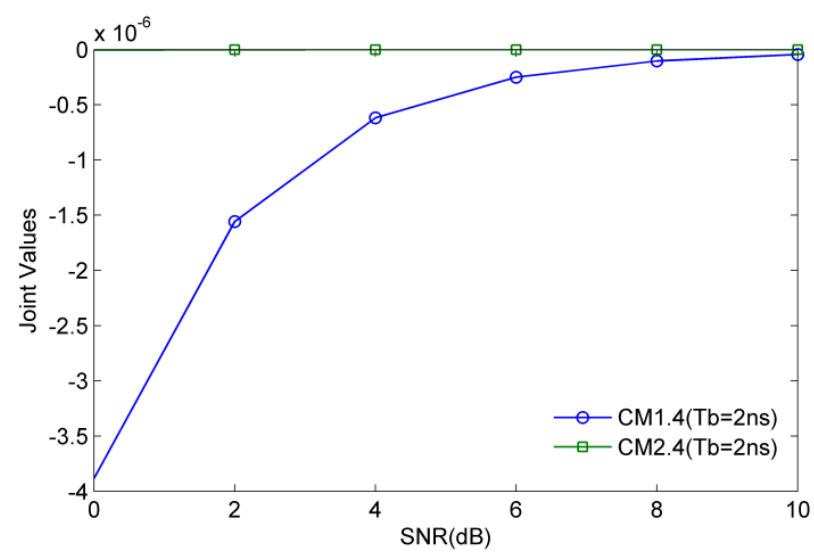

Figure 16. Joint Values with Respect to SNR ( $T b=2 n s)$

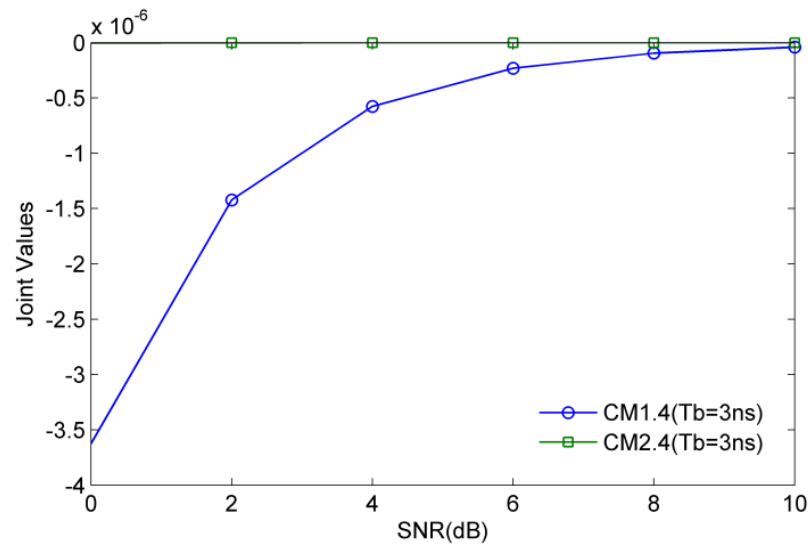

Figure 17. Joint Values with Respect to SNR ( $T b=3 n s)$

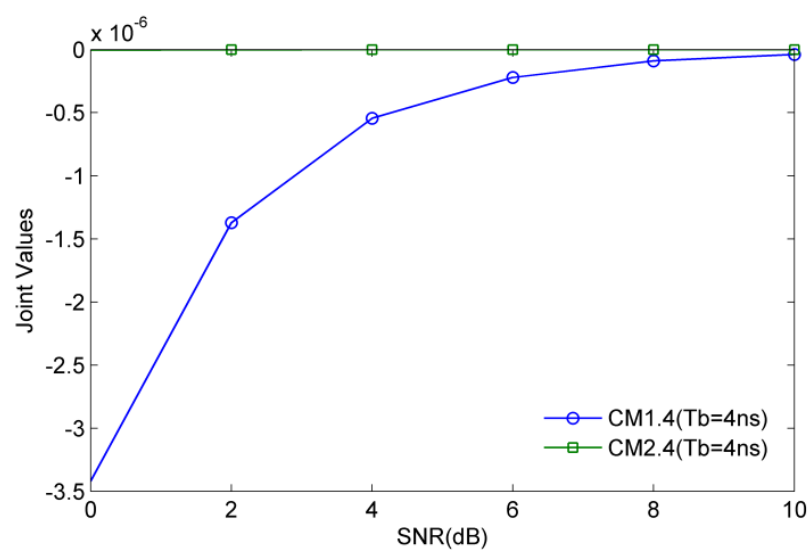

Figure 18. Joint Values with Respect to SNR ( $T b=4 n s)$

From Figures 7-18, results show that joint parameter can identify the LOS and NLOS environments so long as the threshold $\alpha_{M S}$ can be fixed better. The corresponding values are discussed in Table 2.

\section{Conclusion}

In this paper, we presented a novel approach to deal with non-line-of-sight propagation that relies solely on features extracted from the received waveform. This technique does not require formulation of explicit statistical models for features which is based on the 
ratio of minimum slope and standard deviation of energy block of the received signal using ED. Firstly, we use ED TOA Estimation algorithm for the estimation the TOA. Secondly, we characterize the ratio of energy block of the received signal. Finally, we use a threshold test for LOS/NLOS identification. In order to verify effectiveness and practicality of algorithm, so we make a lot of simulations using IEEE 802.15.3c channel models. CM1.1, CM1.2, CM1.3, CM1.4 (residential LOS) and CM2.1, CM2.2, CM2.3, CM2.4 (residential NLOS) channel models from IEEE 802.15.3c standard are employed. Results show that minimum slope can identify LOS and NLOS environments so long as threshold $\alpha_{M S}$ can be fixed bitterly. We developed techniques that are capable of distinguishing LOS/NLOS propagation in NLOS conditions. Our results revealed that the proposed technique outperforms previous parametric techniques from the literature. But here is a question that the method we proposed can't identify the LOS and NLOS environments in office. So in the future, this will be problem which is eager to be solved for us.

\section{Acknowledgments}

The authors would like to thank colleagues from UWB Laboratory in College of Information Science and Engineering, Ocean University of China, for help with obtaining measurement data. This work was supported by Nature Science Foundation of China under Grant No. 60902005, Qingdao International Science and Technology Cooperation Projects of Qingdao under Grant No. 12-1-4-137-hz, and Qingdao Transformation of Scientific and Technological Achievements Guiding Plan (youth special program) under Grant No. 14-2-4-37-jch.

\section{Reference}

[1] L. Zhang, "A fully integrated $60 \mathrm{GHz}$ four channel CMOS receiver with $7 \mathrm{GHz}$ ultra-wide band width for IEEE 802.11ad standard", Communication, China, vol. 11, no. 6, (2014), pp. 42-50.

[2] S. K. Yong and C. C. Chong, "An overview of multi gigabit wireless through millimeter Wave Strategy: Potentials and Technical Challenges", EURASIP Journal on Wireless Communications and Networking, vol. 2007, no. 1, (2007), pp. 1-10.

[3] R. C. Daniels and R. W. Heath, "60 GHz wireless communications: emerging requirements and design recommendations”, IEEE Vehicular Strategy Society, vol. 2, (2007), pp. 41-50.

[4] C. C. Chong, F. M. Peter and Smulders, "60GHz-Millimeter-Wave Radio Principle, Strategy, and News Results", EURASIP Journal on Wireless Communications and Networking, vol. 2007, no. 1, (2007), pp. $1-8$.

[5] S. K. Yong, P. F. Xia and V. G. Alberto, "60-GHz Strategy for Gbps WLAN and WPAN: From Theory to Practice", Beijing: Press of China Machine, (2013).

[6] R. C. Daniels and R. W. Health, " $60 \mathrm{GHz}$ wireless communications: emerging requirements and design recommendations”, IEEE Vehicular Strategy Magazine, vol. 2, no. 3, (2007), pp. 41-50.

[7] M. P. Wylie and J. Holtzman, "The non-line of sight problem in mobile location estimation," in Proceedings of the 5th IEEE International Conference on Universal Personal Communications (ICUPC '96), Cambridge, Mass,USA, vol. 2, (1996), pp. 827-831.

[8] J. Borras, P. Hatrack and N. B. Mandayam, "Decision theoretic framework for NLOS identification," in Proceedings of the 48th IEEE Vehicular Technology Conference (VTC '98), Ottawa, Canada, vol. 2, (1998), pp. 1583-1587.

[9] I. Guvenc, C. C. Chong and F. Watanabe, "NLOS identification and mitigation for UWB localization systems. Wireless Communications and Networking Conference", WCNC 2007, IEEE, (2007), pp. 1571-1576.

[10] M. Heidari, F. O. Akgul and K. Pahlavan, "Identification of the absence of direct path in indoor localization systems", IEEE International Symposium on Personal, Indoor, and Mobile Radio Communications, (2007), pp. 1-6

[11] S. Venkatesh and R. M. Buehrer, "Non-line-of-sight identification in ultra-wideband systems based on received signal statistic", Microwaves, Antennas \& Propagation, IET, vol. 1, no. 6, (2007), pp. 11201130.

[12] H. Shimizu, H. Masui, M. Ishii and K. Sakawa, "LOS and NLOS path-loss and delay characteristics at $3.35 \mathrm{GHz}$ in a residential environment", Antennas and Propagation Society International Symposium, IEEE, vol. 2, (2000), pp. 1142-1145.

[13] I. Güvenç, C. C. Chong, F. Watanabe, H. Inamura, "NLOS identification and weighted least-squares 
localization for UWB systems using multipath channel statistics", EURASIP Journal on Advances in Signal Processing, (2008), p. 4, Article ID 271984.

[14] "IEEE Standard for Information strategy--Local and metropolitan area networks--Specific requirements--Part 15.3: Wireless Medium Access Control (MAC) and Physical Layer (PHY) Specifications for High Rate Wireless Personal Area Networks (WPAN) amendment 2: millimeter-wave-based alternative physical layer extension", IEEE Computer Society, IEEE 802.15.06-0474-00-003c. New York, USA, (2009).

[15] "802.11n-2009-IEEE Standard for Information strategy-- Local and metropolitan area networks--Specific requirements--Part 11: Wireless LAN Medium Access Control (MAC) and Physical Layer (PHY) Specifications Amendment 5: Enhancements for Higher Throughput", IEEE Computer Society, IEEE 978-0-7381-6731-2. New York, USA, (2009).

[16] C. R. Andersonn and T. S. Rappaport, "In-building wideband partition loss measurements at 2.5 and 60GHz", IEEE Transactions on Wireless Communications, vol. 3, no. 3, (2004), pp. 922-928.

[17] S. Collong, G. Zaharia and G. E. Zein, "Influence of the human activity on wide-band characteristics of the $60 \mathrm{GHz}$ indoor radio channel", IEEE Transactions on Wireless Communications, vol. 3, no. 6, (2005), pp. 2396-2406.

[18] A. Maltsev, R. Maslennikov and A. Sevastyanov, "Experimental investigations of 60GHz WLAN systems in office environment", IEEE Journal on Selected Areas in Communications, vol. 27, no. 8, (2009), pp. 1488-1499.

[19] M. G. Sanchez, A. V. Alejos and I. Cuinas, "Comparision of space diversity performance in indoor radio channels at $40 \mathrm{GHz}$ and $60 \mathrm{GHz}$ ", Proc. of European Conference on Wireless Strategy, Amsterdam, (2008).

[20] H. B. Yang, "Channel characteristics and transmission performance for various channel configurations at 60GHz", EURASIP Journal on Wireless Communications and Networking, vol. 2007, no. 1, (2007), pp. 43-43.

\section{Authors}
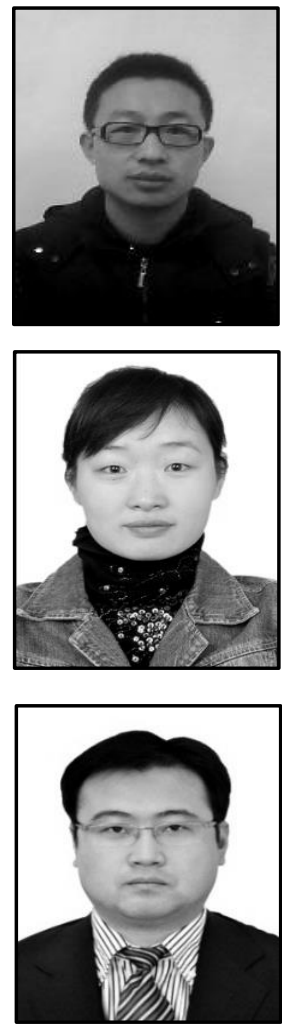

Hao Zhang, he received MBA degree in New York Institute of Technology, American in 2001 and Ph. D. degree in Electrical and Computer Engineering from University of Victoria, Canada in 2004. He was a Project Manager for Microsoft Inc. in United States during January 2000-May 2000. During 2004-2008, he was the Vice President for the United States Gamma Capital Inc. He is now an Adjunct Assistant Professor in Department of Electrical and Computer Engineering. Also he becomes a professor and Ph. D. supervisor in College of Information Science and Engineering from Ocean University of China in 2006. His research concerns ultra-wideband radio systems, $60 \mathrm{GHz}$ wireless communication system and MIMO wireless communication. 
T. Aaron. Gulliver, he received $\mathrm{Ph}$. D. degree in Electrical and Computer Engineering from University of Victoria, Canada in 1989. He is now a professor and $\mathrm{Ph}$. D. supervisor in Department of Electrical and Computer Engineering. In 2002, he becomes a Fellow of the Engineering Institute of Canada, and in 2012 a Fellow of Canadian Academy of Engineering. He is also a senior member of IEEE. His research concerns information theory and communication theory, algebraic coding theory and smart grid and ultra-wideband communication. 